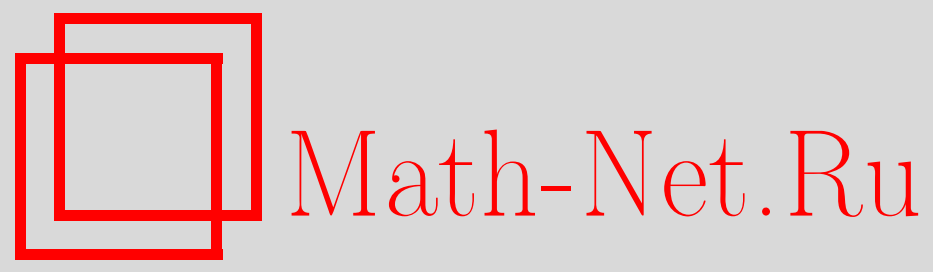

В. Б. Алексеев, А. Д. Поспелов, Сложность умножения в некоторых групповых алгебрах, Дискрет. матем., 2005, том 17, выпуск $1,3-17$

DOI: https://doi.org/10.4213/dm83

Использование Общероссийского математического портала Math-Net.Ru подразумевает, что вы прочитали и согласны с пользовательским соглашением http: //www . mathnet.ru/rus/agreement

Параметры загрузки:

IP: 52.6 .47 .48

26 апреля 2023 г., 05:09:51

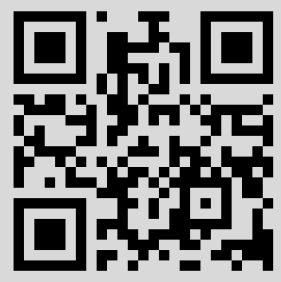




\title{
Сложность умножения в некоторых групповых алгебрах
}

\author{
() 2005 г. В. Б. Алексеев, А. Д. Поспелов
}

\begin{abstract}
В данной работе представлены некоторые результаты из сравнительно новой области теории сложности алгоритмов - алгебраической теории сложности. Рассматривается сложность умножения (билинейная сложность) в групповых алгебрах. В 6-мерной групповой алгебре $\mathbf{C}\left(S_{3}\right)$ над полем комплексных чисел, базисом которой являются подстановки третьего порядка, найден билинейный алгоритм для умножения с мультипликативной сложностью 9 (вместо тривиальных 36) и доказано, что эта оценка неулучшаема. Доказан ряд утверждений о структуре групповой алгебры $\mathbf{C}\left(S_{3}\right)$, в частности, показано, что алгебра $\mathbf{C}\left(S_{3}\right)$ разлагается в прямое произведение алгебры матриц второго порядка и двух одномерных алгебр.

Работа выполнена при подлержке Российского фонда фундаментальных исследований, проект 03-01-00783.
\end{abstract}

\section{1. Основные понятия}

В данной работе представлены некоторые результаты из сравнительно новой области теории сложности алгоритмов - алгебраической теории сложности. Рассматривается сложность умножения в групповых алгебрах (см. определение 2), получаются нетривиальные нижние оценки сложности и приводятся оптимальные по мультипликативной сложности алгоритмы умножения.

В дальнейшем будем считать известными такие алгебраические понятия, как группа, поле и линейное пространство.

Определение 1. Алгеброй (конечномерной) над полем F будем называть произвольное конечномерное линейное пространство $V$ над $\mathbf{F}$ с дополнительно заданной на векторах $V$ операцией умножения, обладающей свойствами ассоциативности и дистрибутивности относительно сложения, а также нейтральным элементом.

Часто в литературе условия ассоциативности и наличия единицы опускаются, если необходимо рассматривать более широкие классы алгебр, однако дистрибутивность относительно имеющегося в алгебре сложения является обязательной, так как связывает оба закона внутренней композиции между собой. В дальнейшем под термином алгебра будем понимать объект, определенный выше.

Определение 2. Пусть $G$ - конечная группа, $G=\left\{g_{1}, g_{2}, \ldots, g_{n}\right\}$ (будем считать, что элементы группы $G$ занумерованы числами от 1 до $n$, причем единичным элементом $G$ является $\left.g_{1}\right)$, и $\mathbf{F}$ - поле. Тогда групповой алгеброй $\mathbf{F}(G)$ группы $G$ над полем $\mathbf{F}$ будем 
называть множество $\mathbf{F}(G)=\left\{\left(\alpha_{1}, \alpha_{2}, \ldots, \alpha_{n}\right)\right\}$ векторов с компонентами из $\mathbf{F}$, на котором операции сложения, умножения на элемент поля и умножение на вектор алгебры заданы следующим образом:

$$
\begin{aligned}
\left(\alpha_{1}, \alpha_{2}, \ldots, \alpha_{n}\right)+\left(\beta_{1}, \beta_{2}, \ldots, \beta_{n}\right)= & \left(\alpha_{1}+\beta_{i}, \alpha_{2}+\beta_{2}, \ldots, \alpha_{n}+\beta_{n}\right), \\
& \forall\left(\alpha_{1}, \alpha_{2}, \ldots, \alpha_{n}\right),\left(\beta_{1}, \beta_{2}, \ldots, \beta_{n}\right) \in \mathbf{F}(G), \\
\gamma \cdot\left(\alpha_{1}, \alpha_{2}, \ldots, \alpha_{n}\right)= & \left(\alpha_{1}, \alpha_{2}, \ldots, \alpha_{n}\right) \cdot \gamma \\
= & \left(\gamma \alpha_{1}, \gamma \alpha_{2}, \ldots, \gamma \alpha_{n}\right), \\
& \forall\left(\alpha_{1}, \alpha_{2}, \ldots, \alpha_{n}\right) \in \mathbf{F}(G), \quad \forall \gamma \in \mathbf{F}, \\
\left(\alpha_{1}, \alpha_{2}, \ldots, \alpha_{n}\right) \cdot\left(\beta_{1}, \beta_{2}, \ldots, \beta_{n}\right)= & \left(\gamma_{1}, \gamma_{2}, \ldots, \gamma_{n}\right), \\
& \forall\left(\alpha_{1}, \alpha_{2}, \ldots, \alpha_{n}\right),\left(\beta_{1}, \beta_{2}, \ldots, \beta_{n}\right) \in \mathbf{F}(G), \\
&
\end{aligned}
$$

где

$$
\gamma_{i}=\sum_{k, l \in\{1, \ldots, n\}: g_{k} g_{l}=g_{i}} \alpha_{k} \beta_{l}, \quad i=1, \ldots, n .
$$

Введенный объект очевидным образом является конечномерным линейным пространством над полем F. Будем ассоциировать $i$-ю компоненту вектора из $V$ с $i$-м элементом группы $G$. Перед дальнейшим изучением групповых алгебр введем следуюшую интерпретацию их элементов: вектор $\left(\alpha_{1}, \alpha_{2}, \ldots, \alpha_{n}\right)$ алгебры $\mathbf{F}(G)$ будем представлять в виде линейной комбинации $\sum_{i=1}^{n} \alpha_{i} g_{i}$. В этом случае введенные определения приобретают наглядность, при этом дистрибутивность умножения относительно сложения в алгебре напрямую следует из дистрибутивности умножения относительно сложения в поле $\mathbf{F}$, ассоциативность умножения - из ассоциативности умножения в группе $G$ (базисом линейного пространства в такой интерпретации будут, очевидно, являться векторы $\left.g_{1}, g_{2}, \ldots, g_{n}\right)$, единичным элементом алгебры будет вектор $g_{1}$.

Определение 3. Базис алгебры $A$ будем называть групповым, если его элементы образуют группу относительно умножения в $A$. Алгебру, в которой существует групповой базис, будем называть групповой алгеброй.

Очевидно, что для любого поля $\mathbf{F}$ и для любой конечной группы $G$ алгебра $\mathbf{F}(G)$ является групповой, при этом групповым базисом в ней будет множество векторов, соответствующих элементам группы $G$.

Введем теперь основные понятия, связанные с алгоритмами умножения в алгебрах. Очевидно, что простейшим алгоритмом умножения, к примеру, в групповой алгебре является следующая последовательность вычислений: для данных векторов $\alpha=\sum_{i=1}^{n} \alpha_{i} g_{i}$, $\beta=\sum_{i=1}^{n} \beta_{i} g_{i}$ сначала вычислить величины $A_{j}^{i}=\alpha_{j} \cdot \beta_{k}$ для $i, j=1,2, \ldots, n$, где $k$ каждый раз выбирается так, что $g_{j} \cdot g_{k}=g_{i}$, то есть $g_{k}=g_{j}^{-1} \cdot g_{i}$, а затем вычислить $\gamma_{i}=\sum_{j=1}^{n} A_{j}^{i}$. При этом $\alpha \cdot \beta=\sum_{i=1}^{n} \gamma_{i} g_{i}$. В этом алгоритме используется $n^{2}$ активных умножений (то есть умножений, в которых оба сомножителя отличны от константы) и $n(n-1)$ сложений. Обобщим эту конструкцию.

Определение 4. Пусть $\mathbf{F}$ - некоторое поле, $U, V, W$ - конечномерные линейные пространства над $\mathbf{F}$, а $\varphi: U \times V \rightarrow W-$ билинейное отображение. Тогда последовательность вида $\left\{f_{1}, g_{1}, h_{1}, \ldots, f_{r}, g_{r}, h_{r}\right\}$, где $f_{i} \in U^{*}, g_{i} \in V^{*}, h_{i} \in W$ называется билинейным алгоритмом длины (ранга) $r$ для $\varphi$, если

$$
\varphi(x, y)=\sum_{t=1}^{r} h_{t} f_{t}(x) g_{t}(y), \quad \forall x \in U, y \in V .
$$


При этом наименьшая длина билинейного алгоритма для $\varphi$ называется билинейной сложностью или рангом $\varphi$ и обозначается $\mathrm{rk} \varphi$. Рангом алгебры $A$ или, иначе, сложностью умножения в алгебре $A$, называется ранг билинейного отображения, соответствующего определению произведения в $A$. Ранг $A$ обозначается через rk $A$.

\section{2. Сложность умножения в $\mathbf{C}\left(S_{2}\right)$}

В качестве примера рассмотрим одну из самых, пожалуй, простых групповых алгебр групповую алгебру подстановок второго порядка над полем комплексных чисел $\mathbf{C}\left(S_{2}\right)$. Рассмотрим группу $S_{2}=\left\{g_{1}, g_{2}\right\}$, таблица умножения в которой задается следуюгцим образом:

\begin{tabular}{c|ll}
$\cdot$ & $g_{1}$ & $g_{2}$ \\
\hline$g_{1}$ & $g_{1}$ & $g_{2}$ \\
$g_{2}$ & $g_{2}$ & $g_{1}$
\end{tabular}

Групповая алгебра в данном случае представляет собой двумерное линейное пространство с базисом $\left\{g_{1}, g_{2}\right\}$. Умножение задается двумя билинейными формами, соответствующими коэффициентам разложения при $g_{1}$ и $g_{2}$ : если необходимо перемножить векторы $\left(x_{1}, x_{2}\right)$ и $\left(y_{1}, y_{2}\right)$ и и $\left(z_{1}, z_{2}\right)=\left(x_{1}, x_{2}\right) \cdot\left(y_{1}, y_{2}\right)$, то

$$
\begin{aligned}
& z_{1}=x_{1} y_{1}+x_{2} y_{2}, \\
& z_{2}=x_{2} y_{1}+x_{1} y_{2} .
\end{aligned}
$$

Как видно, билинейный алгоритм, соответствуюший определению умножения в $\mathbf{C}\left(S_{2}\right)$, имеет длину 4. Ниже предложен билинейный алгоритм длины 2:

$$
\begin{aligned}
P_{1}^{(2)} & =\left(x_{1}+x_{2}\right)\left(y_{1}+y_{2}\right), \\
P_{2}^{(2)} & =\left(x_{1}-x_{2}\right)\left(y_{1}-y_{2}\right), \\
z_{1} & =\frac{1}{2}\left(P_{1}^{(2)}+P_{2}^{(2)}\right), \\
z_{2} & =\frac{1}{2}\left(P_{1}^{(2)}-P_{2}^{(2)}\right) .
\end{aligned}
$$

Таким образом, rk $\mathbf{C}\left(S_{2}\right) \leqslant 2$. С другой стороны, нетрудно видеть, что билинейные формы (1) и (2) линейно независимы и обе имеют ранг 2 . Отсюда следует, что $\operatorname{rk} \mathbf{C}\left(S_{2}\right)$ не может быть равен 1. Таким образом, тривиально установлено, что $\operatorname{rk} \mathbf{C}\left(S_{2}\right)=2$.

\section{3. Сложность умножения в $\mathbf{C}\left(S_{3}\right)$}

Рассмотрим группу $S_{3}=\left\{g_{1}, g_{2}, \ldots, g_{6}\right\}$ подстановок третьего порядка, где $g_{1}$ - тождественная подстановка, $g_{2}=(123), g_{3}=g_{2}^{2}=(132), g_{4}=(12), g_{5}=(13), g_{6}=(23)$. Умножение в ней задается следующей таблицей:

\begin{tabular}{c||lll|lll}
$\cdot$ & $g_{1}$ & $g_{2}$ & $g_{3}$ & $g_{4}$ & $g_{5}$ & $g_{6}$ \\
\hline \hline$g_{1}$ & $g_{1}$ & $g_{2}$ & $g_{3}$ & $g_{4}$ & $g_{5}$ & $g_{6}$ \\
$g_{2}$ & $g_{2}$ & $g_{3}$ & $g_{1}$ & $g_{5}$ & $g_{6}$ & $g_{4}$ \\
$g_{3}$ & $g_{3}$ & $g_{1}$ & $g_{2}$ & $g_{6}$ & $g_{4}$ & $g_{5}$ \\
\hline$g_{4}$ & $g_{4}$ & $g_{6}$ & $g_{5}$ & $g_{1}$ & $g_{3}$ & $g_{2}$ \\
$g_{5}$ & $g_{5}$ & $g_{4}$ & $g_{6}$ & $g_{2}$ & $g_{1}$ & $g_{3}$ \\
$g_{6}$ & $g_{6}$ & $g_{5}$ & $g_{4}$ & $g_{3}$ & $g_{2}$ & $g_{1}$
\end{tabular}


Элемент $g_{1}$ является единицей в $S_{3}$, элементы $g_{1}, g_{2}, g_{3}$ являются четными подстановками, а элементы $g_{4}, g_{5}, g_{6}$ - нечетными подстановками. Заметим, что четные подстановки коммутируют между собой, но не с нечетными подстановками. Умножение задается следуюшими билинейными формами: если $\left(z_{1}, \ldots, z_{6}\right)=\left(x_{1}, \ldots, x_{6}\right) \cdot\left(y_{1}, \ldots, y_{6}\right)$, то

$$
\begin{aligned}
& z_{1}=x_{1} y_{1}+x_{2} y_{3}+x_{3} y_{2}+x_{4} y_{4}+x_{5} y_{5}+x_{6} y_{6}, \\
& z_{2}=x_{1} y_{2}+x_{2} y_{1}+x_{3} y_{3}+x_{4} y_{6}+x_{5} y_{4}+x_{6} y_{5}, \\
& z_{3}=x_{1} y_{3}+x_{2} y_{2}+x_{3} y_{1}+x_{4} y_{5}+x_{5} y_{6}+x_{6} y_{4}, \\
& z_{4}=x_{1} y_{4}+x_{2} y_{6}+x_{3} y_{5}+x_{4} y_{1}+x_{5} y_{2}+x_{6} y_{3}, \\
& z_{5}=x_{1} y_{5}+x_{2} y_{4}+x_{3} y_{6}+x_{4} y_{3}+x_{5} y_{1}+x_{6} y_{2}, \\
& z_{6}=x_{1} y_{6}+x_{2} y_{5}+x_{3} y_{4}+x_{4} y_{2}+x_{5} y_{3}+x_{6} y_{1} .
\end{aligned}
$$

Видно, что алгоритм умножения в $\mathbf{C}\left(S_{3}\right)$, соответствующий определению, имеет мультипликативную сложность 36 . Ниже представлен билинейный алгоритм умножения в $\mathbf{C}\left(S_{3}\right)$, имеющий мультипликативную сложность 9:

$$
\begin{aligned}
P_{1}^{(3)} & =\left(2 x_{1}-x_{2}-x_{3}\right)\left(2 y_{1}-y_{2}-y_{3}\right) \\
P_{2}^{(3)} & =\left(x_{1}-2 x_{2}+x_{3}+x_{4}-2 x_{5}+x_{6}\right)\left(y_{1}-y_{3}+y_{5}-y_{6}\right) \\
P_{3}^{(3)} & =\left(x_{1}-x_{3}+x_{5}-x_{6}\right)\left(-y_{1}+2 y_{2}-y_{3}+y_{4}+y_{5}-2 y_{6}\right) \\
P_{4}^{(3)} & =\left(-x_{1}+2 x_{2}-x_{3}+x_{4}+x_{5}-2 x_{6}\right)\left(y_{1}-2 y_{2}+y_{3}+y_{4}-2 y_{5}+y_{6}\right), \\
P_{5}^{(3)} & =\left(x_{1}+x_{2}-2 x_{3}+x_{4}+x_{5}-2 x_{6}\right)\left(y_{1}-y_{2}-y_{5}+y_{6}\right) \\
P_{6}^{(3)} & =\left(x_{1}-x_{2}-x_{5}+x_{6}\right)\left(-y_{1}-y_{2}+2 y_{3}+y_{4}-2 y_{5}+y_{6}\right) \\
P_{7}^{(3)} & =\left(-x_{1}-x_{2}+2 x_{3}+x_{4}-2 x_{5}+x_{6}\right)\left(y_{1}+y_{2}-2 y_{3}+y_{4}+y_{5}-2 y_{6}\right), \\
P_{8}^{(3)} & =\frac{1}{2}\left(x_{1}+x_{2}+x_{3}-x_{4}-x_{5}-x_{6}\right)\left(y_{1}+y_{2}+y_{3}-y_{4}-y_{5}-y_{6}\right), \\
P_{9}^{(3)} & =\frac{1}{2}\left(x_{1}+x_{2}+x_{3}+x_{4}+x_{5}+x_{6}\right)\left(y_{1}+y_{2}+y_{3}+y_{4}+y_{5}+y_{6}\right) \\
z_{1} & =\frac{1}{3}\left(2 P_{1}^{(3)}-P_{2}^{(3)}+P_{3}^{(3)}+P_{4}^{(3)}-P_{5}^{(3)}+P_{6}^{(3)}+P_{7}^{(3)}+P_{8}^{(3)}+P_{9}^{(3)}\right), \\
z_{2} & =\frac{1}{3}\left(-P_{1}^{(3)}-P_{2}^{(3)}+P_{3}^{(3)}-P_{4}^{(3)}+2 P_{5}^{(3)}-2 P_{6}^{(3)}+P_{8}^{(3)}+P_{9}^{(3)}\right), \\
z_{3} & =\frac{1}{3}\left(-P_{1}^{(3)}+2 P_{2}^{(3)}-2 P_{3}^{(3)}-P_{5}^{(3)}+P_{6}^{(3)}-P_{7}^{(3)}+P_{8}^{(3)}+P_{9}^{(3)}\right), \\
z_{4} & =\frac{1}{3}\left(P_{2}^{(3)}+P_{3}^{(3)}+P_{5}^{(3)}+P_{6}^{(3)}-P_{8}^{(3)}+P_{9}^{(3)}\right) \\
z_{5} & =\frac{1}{3}\left(P_{2}^{(3)}-2 P_{3}^{(3)}+P_{4}^{(3)}-2 P_{5}^{(3)}+P_{6}^{(3)}-P_{7}^{(3)}-P_{8}^{(3)}+P_{9}^{(3)}\right) \\
z_{6} & =\frac{1}{3}\left(-2 P_{2}^{(3)}+P_{3}^{(3)}-P_{4}^{(3)}+P_{5}^{(3)}-2 P_{6}^{(3)}+P_{7}^{(3)}-P_{8}^{(3)}+P_{9}^{(3)}\right) .
\end{aligned}
$$

Отсюда получаем следующее утверждение.

Лемма 1. Справедлива оченка

$$
\operatorname{rk} \mathbf{C}\left(S_{3}\right) \leqslant 9 .
$$

Изучим теперь некоторые свойства алгебры $\mathrm{C}\left(S_{3}\right)$.

Определение 5. Множество $I, I \subseteq A$, где $A$ - алгебра, будем называть левым (правым) идеалом $A$, если $I$ является линейным подпространством $A$ и $a x \in I$ (соответственно $x a \in I)$ для любых элементов $a \in A, x \in I$. Идеал $I$ алгебры $A$ называется двусторонним, если $I$ является и левым, и правым идеалом. 
Определение 6. Левый (правый, двусторонний) идеал $I$ алгебры $A$ называется максимальным, если $I \neq A$ и для любого левого (соответственно правого, двустороннего) идеала $I^{\prime}$ алгебры $A$ выполняется импликация

$$
I \subseteq I^{\prime} \Rightarrow I=I^{\prime}
$$

Лемма 2. Множества

$$
\begin{aligned}
& I_{1}=\left\{\left(\alpha_{1}, \alpha_{2}, \ldots, \alpha_{6}\right) \mid \alpha_{1}+\alpha_{2}+\alpha_{3}+\alpha_{4}+\alpha_{5}+\alpha_{6}=0\right\} \\
& I_{2}=\left\{\left(\alpha_{1}, \alpha_{2}, \ldots, \alpha_{6}\right) \mid \alpha_{1}+\alpha_{2}+\alpha_{3}=\alpha_{4}+\alpha_{5}+\alpha_{6}\right\} \\
& I_{3}=\left\{\left(\alpha_{1}, \alpha_{2}, \ldots, \alpha_{6}\right) \mid \alpha_{1}=\alpha_{2}=\alpha_{3}, \alpha_{4}=\alpha_{5}=\alpha_{6}\right\}
\end{aligned}
$$

являются двусторонними идеалами в $\mathbf{C}\left(S_{3}\right)$, причем $I_{1}, I_{2}, I_{3}$ попарно не вложены друг в друга.

Доказательство. Рассмотрим $I_{1}$. Если мы покажем, что

$$
I_{1} g_{i}=g_{i} I_{1}=I_{1}
$$

для каждого $i \in\{1,2, \ldots, 6\}$, мы, очевидно, докажем, что $I_{1}-$ идеал. Это будет следовать из того, что $I_{1}$ является линейным подпространством $\mathbf{C}\left(S_{3}\right)$ (линейная комбинация векторов, имеющих нулевую сумму координат, также имеет нулевую сумму координат). Но равенства (3) напрямую следуют из того, что умножение на любую подстановку лишь переставляет компоненты вектора, не отождествляя их. Таким образом, нулевая сумма координат вектора не изменяется при умножении на него слева или справа любого другого вектора, то есть $I_{1}$, действительно, является двусторонним идеалом.

Рассмотрим теперь $I_{2}$. Множество $I_{2}$, аналогично $I_{1}$, является линейным подпространством $\mathbf{C}\left(S_{3}\right)$ (это следует из того, что линейная комбинация векторов, имеющих сумму коэффициентов, стоящих при четных подстановках, равную сумме коэффициентов, стоящих при нечетных подстановках, будет обладать тем же свойством). Далее, умножение слева или справа на четную подстановку переводит четные подстановки в четные подстановки, а нечетные подстановки в нечетные подстановки, переставляя некоторым биективным образом коэффициенты при них. Суммы коэффициентов, стоящих при четных подстановках, и суммы коэффициентов, стоящих при нечетных подстановках, очевидно, при этом не меняются. Умножение слева или справа на нечетную подстановку меняет местами коэффициенты, стоящие при четных подстановках и при нечетных подстановках. Суммы коэффициентов, стоящих при четных и нечетных подстановках при этом меняются местами, то есть, в силу равенства, остаются неизменными. В обоих случаях произведение остается в идеале. Наконец, множество $I_{3}$, очевидным образом, является линейным подпространством $\mathbf{C}\left(S_{3}\right)$ и в силу причин, указанных при разборе $I_{2}$, также является идеалом.

Для доказательства попарной невложенности $I_{1}, I_{2}, I_{3}$ приведем примеры векторов $f_{i}$, принадлежащих $I_{i}$, но не принадлежащих $I_{j}, i \neq j, i, j=1,2,3$ :

$$
f_{1}=(1,0,0,-1,0,0), \quad f_{2}=(1,0,0,1,0,0), \quad f_{3}=(1,1,1,0,0,0) .
$$

Лемма доказана.

Определение 7. Покоординатным скалярным произведением в алгебре $A$ над полем C относительно фиксированного базиса $e=\left\{e_{1}, e_{2}, \ldots, e_{n}\right\}$ будем называть билинейную 
форму

$$
(x, y)=\sum_{i=1}^{n} x_{i} \bar{y}_{i}, \quad x, y \in A,
$$

где $x_{i}, y_{i}$ суть коэффициенты разложения векторов $x, y$ по базису $e$, стоящие при $e_{i}$, $i=1,2, \ldots, n$, а $\bar{a}-$ сопряженный элемент для $a$ в поле $\mathbf{C}$.

Построим ортогональные дополнения относительно покоординатного скалярного произведения в групповом базисе, соответствующем $S_{3}$, к $I_{1}, I_{2}, I_{3}$ как к линейным подпространствам пространства $\mathbf{C}\left(S_{3}\right)$. Очевидно, что размерности этих идеалов суть 5,5 и 2 . Тогда базисами $\mathscr{K}_{i}$ ортогональных дополнений идеалов $I_{i}, i=1,2,3$, будут, например,

$$
\begin{aligned}
& \mathscr{K}_{1}=\{(1,1,1,1,1,1)\}, \\
& \mathscr{K}_{2}=\{(1,1,1,-1,-1,-1)\}, \\
& \mathscr{K}_{3}=\{(1,-1,0,0,0,0),(1,0,-1,0,0,0),(0,0,0,1,-1,0),(0,0,0,1,0,-1)\} .
\end{aligned}
$$

Сами ортогональные дополнения идеалов обозначим соответственно $K_{1}, K_{2}, K_{3}$.

Лемма 3. Ортогональное дополнение относительно покоординатного скалярного произведения в произвольном групповом базисе левого (правого) идеала групповой алгебры $A$ является левым (правым) идеалом.

Доказательство. Действительно, пусть $I$ - левый идеал в $A$ (случай, когда $I-$ правый идеал, совершенно аналогичен), $K$ - его ортогональное дополнение, то есть $(x, y)=0$ для всех $x \in I$ и $y \in K$. Рассмотрим произвольный вектор $a \in A$ и докажем, что $a y \in K$ для всех $y \in K$. Очевидно, что если

$$
\delta(i, j, k)= \begin{cases}1, & g_{i} \cdot g_{j}=g_{k}, \\ 0 & \text { в противном случае }\end{cases}
$$

To

$$
x y=\sum_{k=1}^{n} \sum_{i=1}^{n} \sum_{j=1}^{n} x_{i} y_{j} \delta(i, j, k) g_{k},
$$

где $\left\{g_{1}, g_{2}, \ldots, g_{n}\right\}=G$ - некоторый групповой базис алгебры $A$.

Также обозначим через $\pi$ такую подстановку, что

$$
\pi(i)=j \Longleftrightarrow g_{i}^{-1}=g_{j},
$$

при этом $\pi^{-1}=\pi$. Заметим, что $\delta(i, j, k)=\delta(\pi(i), k, j)$.

Пусть теперь $x \in I, y \in K, a \in A$. Если $a=\left(a_{1}, a_{2}, \ldots, a_{n}\right)$, то будем обозначать $a^{\prime}=\left(\bar{a}_{\pi(1)}, \bar{a}_{\pi(2)}, \ldots, \bar{a}_{\pi(n)}\right)$ и $a^{\prime} x=x^{\prime}$. По условию $x^{\prime} \in I$ и $\left(x^{\prime}, y\right)=0$. Нетрудно видеть, что

$$
\begin{aligned}
(x, a y) & =\sum_{k=1}^{n} \sum_{i=1}^{n} \sum_{j=1}^{n} x_{k} \bar{a}_{i} \bar{y}_{j} \delta(i j, k)=\sum_{j=1}^{n} \sum_{i=1}^{n} \sum_{k=1}^{n} \bar{y}_{j} \bar{a}_{i} x_{k} \delta(i, j, k) \\
& =\sum_{j=1}^{n} \sum_{i=1}^{n} \sum_{k=1}^{n} \bar{y}_{j} \bar{a}_{i} x_{k} \delta(\pi(i), k, j)=\sum_{j=1}^{n} \sum_{i=1}^{n} \sum_{k=1}^{n} \bar{y}_{j} \bar{a}_{\pi(i)} x_{k} \delta(i, k, j) \\
& =\sum_{j=1}^{n} \bar{y}_{j} \sum_{i=1}^{n} \sum_{k=1}^{n} a_{i}^{\prime} x_{k} \delta(i, k, j)=\sum_{j=1}^{n} x_{j}^{\prime} \bar{y}_{j}=\left(x^{\prime}, y\right)=0 .
\end{aligned}
$$


Таким образом, действительно, ортогональное дополнение идеала также является идеалом.

Лемма доказана.

Следствие 1. Пусть A- произвольная групповая алгебра, I-двусторонний идеал в $A$, $K$ - ортогональное дополнение к I относительно покоординатного скалярного произведения в некотором групповом базисе. Тогда

$$
x \cdot y=y \cdot x=0, \quad x \in I, \quad y \in K .
$$

Доказательство. Утверждение следствия непосредственно следует из того, что $x \cdot y, y \cdot x \in I, x \cdot y, y \cdot x \in K$ для всех $x \in I$ и $y \in K$, в то время как $I \cap K=\{0\}$.

Замечание 1. Вообще говоря, если нет особых ограничений на структуру алгебры и на определение скалярного произведения, ортогональное дополнение идеала вовсе не обязано также являться идеалом. Действительно, рассмотрим алгебру $T_{n}$ верхних треугольных матриц размера $n \times n$ над произвольным полем $\mathbf{F}$ с естественным базисом $e_{i j}, 1 \leqslant i \leqslant n$, $i \leqslant j \leqslant n$, умножение в котором определяется следующим образом:

$$
e_{i j} \cdot e_{k l}= \begin{cases}e_{i l}, & \text { если } j=k, \\ 0 & \text { в противном случае. }\end{cases}
$$

Скалярное произведение определим как сумму произведений пар коэффициентов при соответствующих векторах разложения по базису $e_{11}, e_{12}, \ldots, e_{n n}$. Тогда для любого $i_{0}$, $1 \leqslant i_{0}<n$, множество векторов

$$
I_{i_{0}}=\left\{\lambda e_{i j} \mid 1 \leqslant i \leqslant i_{0}, n-i_{0}+i \leqslant j \leqslant n, \lambda \in \mathbf{F}\right\},
$$

как нетрудно проверить, порождает идеал в $T_{n}$. Его ортогональным дополнением относительно введенного скалярного произведения является множество векторов

$$
K_{i_{0}}=\left\{\sum_{1 \leqslant i \leqslant n, i \leqslant j<\min \left(n-i_{0}+i, n+1\right)} \alpha_{i j} e_{i j} \mid \alpha_{i j} \in \mathbf{F}\right\} .
$$

Для любого допустимого значения $i_{0}$ вектор $e_{1 n}$ принадлежит $I_{i_{0}}$, а векторы $e_{11}, e_{n n}$ принадлежат $K_{i_{0}}$. Ясно, что $K_{i_{0}}$ в таком случае не является идеалом, так как $e_{1 n} \cdot e_{n n}=e_{1 n}$ и $e_{11} \cdot e_{1 n}=e_{1 n}$.

С другой стороны, в произвольной алгебре всегда можно определить скалярное произведение так, что ортогональное дополнение идеала будет также идеалом (см. главу 11 , $\S 3$, предложение 1 в [4]).

Отметим, что условие вложенности идеалов $I^{\prime} \subseteq I^{\prime \prime}$ равносильно условию обратной вложенности их ортогональных дополнений $K^{\prime} \supseteq K^{\prime \prime}$.

Как известно, пересечение идеалов также является идеалом. Так, рассмотрим идеал $M=I_{1} \cap I_{2}$. Он характеризуется тем, что сумма всех его коэффициентов, стоящих при четных подстановках, равна сумме всех его коэффициентов, стоящих при нечетных подстановках, и равна нулю. Очевидно, что $\operatorname{dim} M=4$. Из определений также ясно, что $I_{3} \cap M=\{0\}, M=K_{3}$ есть ортогональное дополнение к $I_{3}$ относительно покоординатного скалярного произведения и, согласно следствию из леммы $3, f^{\prime} \cdot f^{\prime \prime}=0$, если один из векторов $f^{\prime}, f^{\prime \prime}$ лежит в $I_{3}$, а другой - в $M$.

Докажем теперь следующую теорему, описывающую структуру изучаемой алгебры. 
Теорема 1. Если $\mathbf{C}^{2 \times 2}$ - алгебра матрич второго порядка над полем комплексных чисел, $a \mathbf{C}^{2}$ - алгебра двухкомпонентных векторов с компонентами из $\mathrm{C}$, все операчии в которой производятся покоординатно, то

$$
\mathbf{C}\left(S_{3}\right) \cong \mathbf{C}^{2 \times 2} \times \mathbf{C}^{2},
$$

Доказательство. Рассмотрим следующую систему векторов в $\mathrm{C}\left(S_{3}\right)$ :

$$
\begin{aligned}
e_{11} & =\frac{1}{3}\left(g_{1}-g_{3}+g_{5}-g_{6}\right), \\
e_{12} & =\frac{1}{3}\left(g_{2}-g_{3}+g_{4}-g_{6}\right), \\
e_{21} & =\frac{1}{3}\left(-g_{2}+g_{3}+g_{4}-g_{5}\right), \\
e_{22} & =\frac{1}{3}\left(g_{1}-g_{2}-g_{5}+g_{6}\right), \\
f_{1} & =\frac{1}{6}\left(g_{1}+g_{2}+g_{3}-g_{4}-g_{5}-g_{6}\right), \\
f_{2} & =\frac{1}{6}\left(g_{1}+g_{2}+g_{3}+g_{4}+g_{5}+g_{6}\right) .
\end{aligned}
$$

Легко устанавливается, что $e_{i j} \in M, i, j=1,2, f_{i} \in I_{3}, i=1,2$, а также, что все шесть вышеперечисленных векторов линейно независимы и составляют базис $\mathbf{C}\left(S_{3}\right)$. Из свойств $I_{3}, M$ следует, что (через $\mathscr{L}\left(h_{1}, h_{2}, \ldots, h_{n}\right)$ обозначается линейная оболочка векторов $\left.h_{1}, h_{2}, \ldots, h_{n}\right)$

$$
\begin{aligned}
e_{i j} \cdot f_{k} & =f_{k} \cdot e_{i j}=0, & i, j, k & =1,2, \\
e_{i j} \cdot e_{k l} & \in \mathscr{L}\left(e_{11}, e_{12}, e_{21}, e_{22}\right), & i, j, k, l & =1,2, \\
f_{i} \cdot f_{j} & \in \mathscr{L}\left(f_{1}, f_{2}\right), & i, j & =1,2 .
\end{aligned}
$$

Рассмотрим вложение

$$
\begin{array}{rr}
m_{11}=\left(\begin{array}{ll}
1 & 0 \\
0 & 0
\end{array}\right) \rightarrow e_{11}, & m_{12}=\left(\begin{array}{ll}
0 & 1 \\
0 & 0
\end{array}\right) \rightarrow e_{12}, \\
m_{21}=\left(\begin{array}{ll}
0 & 0 \\
1 & 0
\end{array}\right) \rightarrow e_{21}, & m_{22}=\left(\begin{array}{ll}
0 & 0 \\
0 & 1
\end{array}\right) \rightarrow e_{22}, \\
c_{1}=(1,0) \longrightarrow f_{1}, & c_{2}=(0,1) \longrightarrow f_{2} .
\end{array}
$$

Нетрудно убедиться в том, что это есть изоморфное вложение алгебры матриц $\mathbf{C}^{2 \times 2}$ в $M$ и $\mathrm{C}^{2}$ в $I_{3}$.

Из всего вышесказанного следует, что в С $\left(S_{3}\right)$ можно перейти к такому базису, в котором множество базисных векторов разбивается на две непересекающиеся группы $B_{1}$ и $B_{2}$ так, что умножение локализовано внутри этих групп, причем

$$
\mathscr{L}\left(B_{1}\right) \cong \mathbf{C}^{2 \times 2}, \quad \mathscr{L}\left(B_{2}\right) \cong \mathbf{C}^{2} .
$$

Это по определению декартова произведения алгебр означает, что выполняется (4).

Теорема доказана.

Приведем без доказательства следующую теорему, принадлежащую А. Алдеру и Ф. Штрассену (см. [1]).

Теорема 2. Для произвольной ассочиативной алгебры $A$

$$
\text { rk } A \geqslant 2 \operatorname{dim} A-t(A),
$$

где $t(A)$ - число максимальных двусторонних идеалов А. 
Будем называть алгебру $A$ алгеброй минимального ранга, если вместо (5) выполняется равенство

$$
\text { rk } A=2 \operatorname{dim} A-t(A) .
$$

Левый (правый, двусторонний) идеал I называется нильпотентным, если для некоторого целого числа $n$ выполняется равенство $I^{n}=\{0\}$. Сумма всех нильпотентных левых идеалов алгебры $A$ называется радикалом $A$ и обозначается $\operatorname{rad} A$. Известен следующий факт (см. [3]).

Предложение 1. Радикал А содержится в любом максимальном двустороннем идеале А.

Алгебра $A$ называется полупростой, если $\operatorname{rad} A=\{0\}$. Алгебра $A$ называется простой, если она не содержит двусторонних идеалов, кроме нулевого и $A$.

Будем обозначать множество обратимых элементов алгебры $A$ через $A^{\times}$. Алгебра $D$ называется алгеброй с делением, если $D^{\times}=D \backslash\{0\}$. Алгебра $A$ называется локальной, если фактор-алгебра $A / \operatorname{rad} A$ является алгеброй с делением, и $A$ называется основной, если $A / \operatorname{rad} A$ является прямым произведением алгебр с делением. Будем называть алгебру $A$ над полем $\mathbf{F}$ алгеброй Блезера (см. [2]), если $A / \operatorname{rad} A \cong \mathbf{F}^{t}$ для некоторого $t$.

Для дальнейшей характеризации структуры $\mathbf{C}\left(S_{3}\right)$ приведем без доказательства следующую теорему, характеризующую все алгебры минимального ранга над произвольными полями и принадлежащую М. Блезеру (см. [2]).

Теорема 3. Алгебра А над произвольным полем $k$ является алгеброй минимального ранга тогда и только тогда, когда

$$
A \cong C_{1} \times \ldots \times C_{s} \times k^{2 \times 2} \times \ldots \times k^{2 \times 2} \times B,
$$

где поле $k$ входит в произведение и раз, $C_{1}, \ldots, C_{s}$ суть локальные алгебры минимального ранга, для которых $\operatorname{dim}\left(C_{\sigma} / \operatorname{rad} C_{\sigma}\right) \geqslant 2$, то есть

$$
C_{\sigma} \cong k[X] /\left(p_{\sigma}(X)^{d_{\sigma}}\right)
$$

для некоторых неприводимых полиномов $p_{\sigma}$ над полем $k, \operatorname{deg} p_{\sigma} \geqslant 2, d_{\sigma} \geqslant 1 u$ $\sharp k \geqslant 2 \operatorname{dim} C_{\sigma}-2$, а B есть алгебра Блезера минимального ранга. Любое из чисел $s$, и может быть нулем, а множитель В является необязательным.

Максимальными двусторонними идеалами $\mathbf{C}^{2}$ являются линейные оболочки векторов $(1,0)$ и $(0,1)$. Отсюда следует, что $\operatorname{rad} \mathbf{C}^{2}=\{0\}$, так как радикал алгебры содержится в пересечении максимальных двусторонних идеалов этой алгебры. Но тогда $\mathbf{C}^{2} / \mathrm{rad}^{2} \cong \mathbf{C}^{2}$, откуда, по определению, $\mathbf{C}^{2}$ является алгеброй Блезера. Используя теорему 3 , а также учитывая (4) и вышесказанное, получаем следующую теорему.

Теорема 4. Алгебра $\mathbf{C}\left(S_{3}\right)$ является алгеброй минимального ранга.

Покажем, что $I_{1}, I_{2}, I_{3}$ суть все максимальные идеалы $\mathbf{C}\left(S_{3}\right)$.

Лемма 4. Каждый двусторонний идеал I алгебры С $\left(S_{3}\right)$ вложен в один из идеалов $I_{1}, I_{2}, I_{3}$.

Доказательство. Рассмотрим $K$, ортогональное дополнение $I$. Будем существенно пользоваться тем, что $K$ - также двусторонний идеал. Возможны два случая. 
В первом случае в $K$ есть вектор с ненулевой суммой координат

$$
x=\left(x_{1}, x_{2}, \ldots, x_{6}\right) \text {. }
$$

Тогда рассмотрим вектор $x\left(g_{1}+g_{2}+\ldots+g_{6}\right)$, у которого каждая координата равна сумме всех координат $x$, так что все координаты равны. Но тогда $K_{1} \subseteq K$ и, согласно сделанному ранее замечанию, $I \subseteq I_{1}$.

Во втором случае все векторы $K$ имеют нулевую сумму координат. Тогда возможны два варианта.

В первом варианте в $K$ есть вектор $x$ с ненулевой суммой коэффициентов при четных подстановках. Тогда рассмотрим вектор

$$
y=x\left(g_{1}+g_{2}+g_{3}\right), \quad y \in K .
$$

Вектор $y$ имеет равные коэффициенты $y_{1}$ при всех четных подстановках, причем они ненулевые, так как равны сумме всех коэффициентов четных подстановок $x$, и равные коэффициенты $y_{2}$ при всех нечетных подстановках, равные сумме всех коэффициентов нечетных подстановок $x$. При этом сумма коэффициентов $y$ равна утроенной сумме ко'фффициентов $x$ и равна 0 . Поэтому $y_{1}=-y_{2}$ и $y$ составляет базис $K_{2}$. Тогда $K_{2} \subseteq K$ и, согласно сделанному замечанию, $I \subseteq I_{2}$.

Ясно, что аналогичные рассуждения справедливы, если отлична от нуля сумма коэффициентов при нечетных подстановках. В этом случае надо рассмотреть вектор $y^{\prime}=g_{4} y$, у которого коэффициенты при четных и нечетных подстановках поменяются местами.

Во втором варианте все векторы в $K$ имеют нулевую сумму коэффициентов при четных и при нечетных подстановках по отдельности, при этом в $K$ присутствует вектор вида

$$
x=(a, b,-a-b, c, d,-c-d),
$$

причем среди комплексных чисел $a, b, c, d$ хотя бы одно отлично от нуля. Если отлично от нуля одно из чисел $c, d$, то будем рассматривать вектор $y=g_{4} x$, в противном случае положим $y=x$. Введем обозначение

$$
z=y+g_{2} y g_{3}+g_{3} y g_{2}, \quad z \in K .
$$

Существенно, что вектор $z$ имеет хотя бы один ненулевой коэффициент при четной подстановке и все его коэффициенты при нечетных подстановках суть нули (это следует из того, что четные подстановки коммутируют друг с другом, но не с нечетными подстановками, см. таблицу умножения в разделе 3). Коэффициенты при четных подстановках у $z$ суть соответствующие утроенные коэффициенты при четных подстановках у $y$. В то же время, если $g_{i}-$ нечетная подстановка, то $g_{i}, g_{2} g_{i} g_{3}, g_{3} g_{i} g_{2}$ - три различные нечетные подстановки. Таким образом,

$$
z=(f, g,-f-g, 0,0,0)
$$

где $|f|+|g| \neq 0$. В $K$ лежат также следующие два вектора:

$$
\begin{aligned}
z^{\prime} & =g_{2} z=(g,-f-g, f, 0,0,0), \\
z^{\prime \prime} & =g_{4} z g_{6}=(g, f,-f-g, 0,0,0) .
\end{aligned}
$$

Если $f= \pm g$, то $z$ и $z^{\prime}$ линейно независимы. В противном случае, как нетрудно проверить, линейно независимы $z$ и $z^{\prime \prime}$. Таким образом, в $K$ обязательно присутствуют два линейно 
независимых вектора $y_{1}, y_{2}$ с нулевыми коэффициентами при нечетных подстановках. Домножениями на некоторую нечетную подстановку, например, на $g_{4}$, получаем еще два линейно независимых между собой и с $y_{1}, y_{2}$ вектора $y_{3}, y_{4}$ с нулевыми коэффициентами при четных подстановках.

Таким образом, $K$ содержит линейное подпространство размерности 4, все элементы которого имеют нулевые суммы коэффициентов при четных подстановках и нулевые суммы коэффищиентов при нечетных подстановках. Но таким подпространством и является $K_{3}$, следовательно, $K_{3} \subseteq K$ и, согласно сделанному выше замечанию, $I \subseteq I_{3}$.

Лемма доказана.

Таким образом, в силу теоремы 2 можно заключить, что имеет место следующая лемма.

Лемма 5. Справедлива оченка

$$
\operatorname{rk} \mathbf{C}\left(S_{3}\right) \geqslant 9
$$

Сравнивая результаты лемм 1 и 5, получаем следующую теорему.

Теорема 5. Справедливо равенство

$$
\operatorname{rk} \mathbf{C}\left(S_{3}\right)=9
$$

\section{4. Дополнительные сведения о $\mathbf{C}\left(S_{3}\right)$}

В доказательстве теоремы 1 было построено изоморфное вложение алгебры матриц $\mathbf{C}^{2 \times 2}$ в групповую алгебру $\mathbf{C}\left(S_{3}\right)$. При этом изоморфным образом $\mathbf{C}^{2 \times 2}$ в $\mathbf{C}\left(S_{3}\right)$ служил двусторонний идеал $M$. Оказывается, что этот результат теоремы 1 допускает некоторое усиление, а именно, справедлива следуюшая теорема.

Теорема 6. Алгебра $\mathrm{C}\left(S_{3}\right)$ содержит единственную подалгебру, изоморфную алгебре матрич $\mathbf{C}^{2 \times 2}$. Этой подалгеброй является $M$.

Доказательство. Для доказательства утверждения теоремы нам потребуются две следующие леммы.

Лемма 6. В алгебре $\mathbf{C}\left(S_{3}\right)$ элемент $x, x \neq 0, x=\left(x_{1}, x_{2}, x_{3}, x_{4}, x_{5}, x_{6}\right)$, обладает свойством

$$
x \cdot x=x
$$


тогда и только тогда, когда он равен одному из элементов $u_{1}, \ldots, u_{11}$, где

$$
\begin{aligned}
& u_{1}=\left(\frac{2}{3},-\frac{1}{3},-\frac{1}{3}, 0,0,0\right), \\
& u_{2}=\left(\frac{1}{3}, x,-\frac{1}{3}-x, y, z,-y-z\right), \\
& u_{3}=(1,0,0,0,0,0), \\
& u_{4}=\left(\frac{1}{3}, \frac{1}{3}, \frac{1}{3}, 0,0,0\right), \\
& u_{5}=\left(\frac{2}{3}, x, \frac{1}{3}-x, y, z,-y-z\right), \\
& u_{6}=\left(\frac{5}{6},-\frac{1}{6},-\frac{1}{6},-\frac{1}{6},-\frac{1}{6},-\frac{1}{6}\right), \\
& u_{7}=\left(\frac{1}{6}, \frac{1}{6}, \frac{1}{6},-\frac{1}{6},-\frac{1}{6},-\frac{1}{6}\right), y z+z^{2}=x^{2}-\frac{1}{3} x+\frac{1}{9}, \\
& u_{8}=\left(\frac{1}{2}, x,-x, y, z,-\frac{1}{2}-y-z\right), \\
& u_{9}=\left(\frac{5}{6},-\frac{1}{6},-\frac{1}{6}, \frac{1}{6}, \frac{1}{6}, \frac{1}{6}\right), \\
& u_{10}=\left(\frac{1}{6}, \frac{1}{6}, \frac{1}{6}, \frac{1}{6}, \frac{1}{6}, \frac{1}{6}\right), \\
& u_{11}=\left(\frac{1}{2}, x,-x, y, z, \frac{1}{2}-y-z+z^{2}+\frac{1}{2} y+\frac{1}{2} z=x^{2},\right.
\end{aligned}
$$

Доказательство. Рассмотрим разложение (4) и перейдем в $\mathbf{C}\left(S_{3}\right)$ к базису $\left\{e_{11}, e_{12}, e_{21}\right.$, $\left.e_{22}, f_{1}, f_{2}\right\}$, построенному в ходе доказательства теоремы 1 . Это означает, что каждому вектору $x, x \in \mathbf{C}\left(S_{3}\right)$, можно взаимно однозначно поставить в соответствие пару $\left(m_{x}, c_{x}\right)$, где $m_{x} \in \mathbf{C}^{2 \times 2}, c_{x} \in \mathbf{C}^{2}$, так, что вектору $x_{1} x_{2}$ будет соответствовать пара

$$
\left(m_{x_{1} x_{2}}, c_{x_{1} x_{2}}\right)=\left(m_{x_{1}} m_{x_{2}}, c_{x_{1}} c_{x_{2}}\right)
$$

Это, в свою очередь, значит, что условие $x \cdot x=x$ выполняется тогда и только тогда, когда $m_{x} m_{x}=m_{x}$ и $c_{x} c_{x}=c_{x}$. Таким образом, необходимо и достаточно охарактеризовать все элементы $\mathbf{C}^{2 \times 2}$ и $\mathbf{C}^{2}$, обладающие указанным свойством.

Рассмотрим произвольный вектор $z=(x, y), z \in \mathbf{C}^{2}$. Для него это условие равносильно выполнению равенств $x^{2}=x, y^{2}=y$. Отсюда немедленно следует, что $x, y \in\{0,1\}$ и элемент $z$ в $\mathbf{C}^{2}$ обладает свойством $z \cdot z=z$ тогда и только тогда, когда он совпадает с одним из векторов

$$
z_{1}=(0,0), \quad z_{2}=(1,1), \quad z_{3}=(1,0), \quad z_{4}=(0,1)
$$

Рассмотрим теперь произвольную матрицу из $\mathbf{C}^{2 \times 2}$. Пусть

$$
m=\left(\begin{array}{ll}
a & b \\
c & d
\end{array}\right) .
$$


Тогда условие $m \cdot m=m$ равносильно выполнению следующих четырех равенств:

$$
\begin{aligned}
& a^{2}+b c=a, \\
& a b+b d=b, \\
& a c+c d=c, \\
& b c+d^{2}=d .
\end{aligned}
$$

Возможны два варианта.

В первом варианте $a+d \neq 1$. Тогда из (7), (8) следует, что $b=c=0$, а из (6), (9) следует, что $a, d \in\{0,1\}$. Отсюда получаем, что допустимы две матрицы

$$
m_{1}=\left(\begin{array}{ll}
1 & 0 \\
0 & 1
\end{array}\right), \quad m_{2}=\left(\begin{array}{ll}
0 & 0 \\
0 & 0
\end{array}\right) \text {. }
$$

Во втором варианте $a+d=1$. Тогда уравнения (7), (8) выполняются автоматически, а соотношения (6) и (9) дают необходимое и достаточное условие того, что $m \cdot m=m$, состоящее в том, что

$$
a^{2}+b c=a
$$

Таким образом, получаем трехпараметрическое квадратичное многообразие матриц

$$
m_{3}=\left(\begin{array}{cc}
a & b \\
c & 1-a
\end{array}\right), \quad a^{2}+b c=a .
$$

Перебирая всевозможные комбинации векторов $z, z \cdot z=z, z \in \mathbf{C}^{2}$, и матриц $m$, $m \cdot m=m, m \in \mathbf{C}^{2 \times 2}$, получаем двенадцать типов векторов $\mathbf{C}\left(S_{3}\right)$, удовлетворяющих условию леммы. В число этих двенадцати попадает также нулевой вектор, порождаемый парой $\left(z_{1}, m_{2}\right)$, который интереса не представляет и поэтому не рассматривается. Нетрудно проверить, что следующие пары матриц и векторов из $\mathbf{C}^{2}$ порождают следующие типы векторов в $\mathbf{C}\left(S_{3}\right)$ (с точностью до переобозначения параметров):

$$
\begin{array}{ll}
\left(z_{1}, m_{1}\right) \rightarrow u_{1}, & \left(z_{3}, m_{1}\right) \rightarrow u_{6}, \\
\left(z_{1}, m_{2}\right) \rightarrow 0, & \left(z_{3}, m_{2}\right) \rightarrow u_{7}, \\
\left(z_{1}, m_{3}\right) \rightarrow u_{2}, & \left(z_{3}, m_{3}\right) \rightarrow u_{8}, \\
\left(z_{2}, m_{1}\right) \rightarrow u_{3}, & \left(z_{4}, m_{1}\right) \rightarrow u_{9}, \\
\left(z_{2}, m_{2}\right) \rightarrow u_{4}, & \left(z_{4}, m_{2}\right) \rightarrow u_{10}, \\
\left(z_{2}, m_{3}\right) \rightarrow u_{5}, & \left(z_{4}, m_{3}\right) \rightarrow u_{11}
\end{array}
$$

Лемма доказана.

Лемма 7. В алгебре $\mathrm{C}\left(S_{3}\right)$ элемент $u_{0}, u_{0}=\left(u_{1}, u_{2}, u_{3}, u_{4}, u_{5}, u_{6}\right)$, обладает свойством

$$
u_{0} \cdot u_{0}=0
$$

тогда и только тогда, когда он представим в виде

$$
u_{0}=(0, x,-x, y, z,-y-z), \quad x^{2}=y^{2}+y z+z^{2} .
$$


Доказательство. Аналогично доказательству предыдущей леммы воспользуемся разложением (4). Единственным нильпотентным вектором $\mathbf{C}^{2}$ является нулевой вектор, следовательно, все множество удовлетворяющих условию леммы векторов $\mathbf{C}\left(S_{3}\right)$ описывается такими ненулевыми матрицами $m, m \in \mathbf{C}^{2 \times 2}$, что $m \cdot m=0$.

Снова рассмотрим произвольную матрицу из $\mathbf{C}^{2 \times 2}$. Пусть

$$
m=\left(\begin{array}{ll}
a & b \\
c & d
\end{array}\right) .
$$

Тогда условие $m \cdot m=0$ равносильно выполнению следующих четырех условий:

$$
\begin{aligned}
& a^{2}+b c=0, \\
& a b+b d=0, \\
& a c+c d=0, \\
& b c+d^{2}=0 .
\end{aligned}
$$

Возможны два варианта.

В первом варианте $a+d \neq 0$. Тогда из (11), (12) следует, что $b=c=0$, и из (10), (13) получаем, что $a=d=0$, что противоречит начальному предположению. Это означает, что все интересующие нас матрицы имеют нулевой след.

Во втором случае $a+d=0$. Тогда равенства (11), (12) выполняются автоматически, а $(10),(13)$ дают необходимое и достаточное условие того, что $m \cdot m=0$.

Таким образом, множество матриц $m, m \in \mathbf{C}^{2 \times 2}, m \cdot m=0$, определяется однозначно:

$$
m_{0}=\left(\begin{array}{rr}
a & b \\
c & -a
\end{array}\right), \quad a^{2}+b c=0 .
$$

Нетрудно проверить, что с точностью до переобозначения переменных это трехпараметрическое квадратичное многообразие матриц порождает семейство векторов $u_{0}$.

Лемма доказана.

Из леммы 7 следует, что размерность подпространства $M_{0}$, порожденного векторами, в квадрате дающими 0 , не превосходит 3 . Покажем, что оно в точности имеет размерность 3. Рассмотрим три таких вектора, удовлетворяющие лемме 7:

$$
\begin{aligned}
& e_{1}^{\prime}=(0,1,-1,1,0,-1), \quad x=1, \quad y=1, \quad z=0, \\
& e_{2}^{\prime}=(0,-1,1,1,0,-1), \quad x=-1, \quad y=1, \quad z=0 \text {, } \\
& e_{3}^{\prime}=(0,1,-1,0,1,-1), \quad x=1, \quad y=0, \quad z=1 \text {. }
\end{aligned}
$$

Они линейно независимы, что очевидно, если через них выразить более наглядный базис этого подпространства:

$$
\begin{array}{ll}
e_{1}=(0,1,-1,0,0,0), & e_{1}=\frac{1}{2}\left(e_{1}^{\prime}-e_{2}^{\prime}\right), \\
e_{2}=(0,0,0,1,0,-1), & e_{2}=\frac{1}{2}\left(e_{1}^{\prime}+e_{2}^{\prime}\right), \\
e_{3}=(0,0,0,0,1,-1), & e_{3}=e_{2}^{\prime}+e_{3}^{\prime}-e_{2} .
\end{array}
$$

В алгебре матриц $\mathbf{C}^{2 \times 2}$ существуют три линейно независимые матрицы, в квадрате дающие нулевую матрицу. Это

$$
m_{1}=\left(\begin{array}{ll}
0 & 1 \\
0 & 0
\end{array}\right), \quad m_{2}=\left(\begin{array}{ll}
0 & 0 \\
1 & 0
\end{array}\right), \quad m_{3}=\left(\begin{array}{ll}
1 & -1 \\
1 & -1
\end{array}\right) .
$$


Образы $m_{i}$ должны быть линейно независимы и в квадрате давать нулевой вектор. Тогда они составляют базис $M_{0}$ и как-то выражаются через векторы $e_{1}, e_{2}, e_{3}$. Это означает, что прообразом $M_{0}$ является множество матриц вида

$$
m=\left(\begin{array}{rr}
x_{1} & x_{2} \\
x_{3} & -x_{1}
\end{array}\right)
$$

то есть матриц с нулевым следом. Среди матриц такого вида существует множество матриц, которые в квадрате дают ненулевую матрицу $m^{\prime}$ такую, что $\left(m^{\prime}\right)^{2}=m^{\prime}$, причем $m^{\prime}$ единственна и равна единичной матрице. Действительно, для любой матрицы $m$ указанного вида

$$
m^{2}=m^{\prime}=\left(\begin{array}{cc}
x_{1}^{2}+x_{2} x_{3} & 0 \\
0 & x_{1}^{2}+x_{2} x_{3}
\end{array}\right)=\left(\begin{array}{ll}
a & 0 \\
0 & a
\end{array}\right)=\left(\begin{array}{cc}
a^{2} & 0 \\
0 & a^{2}
\end{array}\right)=\left(m^{\prime}\right)^{2} .
$$

Отсюда $a=1$ и $m^{\prime}=E$. Таким образом, квадратом некоторого элемента из $M_{0}$ является один из векторов $u_{1}, \ldots, u_{11}$ из леммы 6 . Возьмем произвольный вектор $x=\left(0, x_{1},-x_{1}, x_{2}, x_{3},-x_{2}-x_{3}\right)$ из $M_{0}$ и рассмотрим его квадрат

$$
\begin{array}{r}
\left(x_{1} g_{2}-x_{1} g_{3}+x_{2} g_{4}+x_{3} g_{5}-\left(x_{2}+x_{3}\right) g_{6}\right)\left(x_{1} g_{2}-x_{1} g_{3}+x_{2} g_{4}+x_{3} g_{5}-\left(x_{2}+x_{3}\right) g_{6}\right) \\
=\left(-x_{1}^{2}+x_{2}^{2}+x_{3}^{2}+x_{2} x_{3}\right)\left(2 g_{1}-g_{2}-g_{3}\right)=\lambda\left(2 g_{1}-g_{2}-g_{3}\right) .
\end{array}
$$

Если $x \cdot x \neq 0$, то и $\lambda \neq 0$. Отсюда следует, что образом $E$ является $u_{1}$.

Заметим далее, что единичная матрица $E$ дополняет $m_{1}, m_{2}, m_{3}$ до базиса $\mathbf{C}^{2 \times 2}$. Таким образом, изоморфное вложение $\mathbf{C}^{2 \times 2}$ в $\mathbf{C}\left(S_{3}\right)$, если оно существует, определено однозначно:

$$
\begin{aligned}
E & \longrightarrow u_{1}, \\
\left\{m_{1}, m_{2}, m_{3}\right\} & \longrightarrow M_{0} .
\end{aligned}
$$

В то же время нетрудно проверить, что изоморфное вложение, предложенное в доказательстве теоремы 1 , удовлетворяет этим условиям. Осталось заметить, что $M$ является суммой подпространства $M_{0}$ и линейной оболочки вектора $u_{1}$.

Теорема доказана.

Следствие 2. Изоморфное вложение $\mathbf{C}^{2 \times 2}$ в $\mathbf{C}\left(S_{3}\right)$ определено однозначно с точностью до автоморфизмов самой алгебры матрии $\mathbf{C}^{2 \times 2}$.

\section{Список литературы}

1. Alder A., Strassen V., On the algorithmic complexity of associative algebras. Theoret. Comput. Sci. (1981) 15, 201-211.

2. Bläser M., Algebras of minimal rank over arbitrary fields. SIIM Techn. Rep., May 10, 2002.

3. Ван дер Варден Б. Л., Алгебра. Наука, Москва, 1979.

4. Винберг Э. Б., Курс алгебры. Факториал Пресс, Москва, 2001.

Статья поступила 26.04.2004. 